\title{
Delegating Decisions to Experts
}

Hao Li; Wing Suen

The Journal of Political Economy; Feb 2004; 112, 1; ABI/INFORM Global pg. S311

\section{Delegating Decisions to Experts}

\section{Hao Li}

University of Toronto

\section{Wing Suen}

Universily of Hong Kong

\begin{abstract}
We present a model of delegation with self-interested and privately informed experts. A team of experts with extreme but opposite biases is acceptable to a wide range of decision makers with diverse preferences, but the value of expertise from such a team is low. A decision maker wants to appoint experts who are less partisan than he is in order to facilitate information pooling by the expert team. Selective delegation, either by controlling the decision-making process or by conditioning the delegation decision on his own information, is an effective way for the decision maker to safeguard own interests while making use of expert information.
\end{abstract}

A good executive is the one who understands how to recruit people and how to delegate. [George W. Bush; January 2, 2001, news conference announcing his Cabinet]

This paper is dedicated to the memory of Sherwin Rosen. It grew out of discussions with him in writing a companion paper ( $\mathrm{Li}$, Rosen, and Suen 2001) and would not have been possible without his insights and encouragement. We thank an anonymous referee and the editor for detailed comments and suggestions on an earlier draft. We also received valuable comments from Doug Allen, William Chan, Julian Jamison, Edward Lazcar, Steve Tadelis, and Keith Wong. The work described in this paper was supported by a grant from the Research Grants Council of Hong Kong (project no. HKU 7132/01H). 


\section{Introduction}

Consider a president who has to decide whether to approve funding for a research project on a new military technology. The defense secretary has better information about the effectiveness and the potential of the technology than the president does, but he is also biased toward adoption because he is interested in expanding the defense department. Will the president delegate the decision to the secretary? The answer depends on the president's default choice if he is to make the decision himself. Suppose that the president is predisposed to adopt the project on the basis of his own prior. Delegating the decision to the defense secretary will change the outcome when the secretary recommends rejection. Since he is biased toward adoption, the secretary recommends rejection only when receiving strong negative signals about the project. Therefore, the president cannot lose by delegating the decision to the secretary and often gains from the secretary's expert information. If instead the president will reject the project on the basis of his own prior, he has to worry about the tendency of the defense secretary to adopt on the basis of relatively weak signals about the project's usefulness if he delegates the decision. Delegation will not occur if the secretary is sufficiently biased toward adoption.

In this paper we discuss delegation mechanisms that can be used by decision makers to mitigate the agency problem of information manipulation in nonmarket organizations. We show that an expert with more extreme preferences has a greater chance of being delegated the decision by decision makers with similar but less extreme preferences. Thus more extreme experts are more influential in an environment in which experts are recruited before their private information is gathered to help out multiple decision makers or the same decision maker on different decisions. For example, a cabinet member is selected to advise the president on many decisions, and on each individual decision the biases of the member and the president dictate whether the president makes the choice without consulting the expert and delegating the choice to the cabinet member. When a Republican president picks an extremely conservative cabinet member, he can safely delegate more decisions and make use of the member's expertise. Unfortunately, there is a trade-off between the influence and the value of expertise: an expert with more extreme preferences is more likely to be delegated different decisions, but his expertise is less valuable to the decision maker on a given decision. To continue the example above, a strongly conservative cabinet member is more influential with a conservative president, but precisely because of his strong bias, on most decisions the member will make the same choices that the uninformed president would, which reduces the value of his expertise. 
A key to reducing manipulation is to recruit the right expert. Obviously the ideal expert on a given decision is one with preferences identical to those of the decision maker. However, if the decision maker is to appoint a delegate to sit on a committee of experts that will make the decision, the best choice is not to appoint a clone. For example, suppose that committee members are prone to raise taxes whereas the decision maker is a tax-cutting Republican. If he picks an equally staunch Republican as his delegate, the committee will be beset by great conflicts and expert information will be poorly utilized. The decision maker can do better by appointing someone whose preferences are somewhere between his own and those of the other committee members. Although this delegate will not vote exactly the way the decision maker would, there will be less manipulation and the quality of the committee decision will be higher, benefiting the decision maker.

An informed decision maker can condition the delegation decision on his own private signals. Consider again the example of the president and the defense secretary. Since the secretary is prone to adopt the military project, the president may be tempted to reject the project on his own and delegate the decision only when his own information provides insufficient evidence against adoption. But in delegating the decision, the president reveals part of his private information and gives the secretary a greater tendency to adopt the project on the basis of weak signals. This will in turn make the president more cautious and less willing to delegate. A better delegation mechanism for the president is to retain control of both adoption and rejection decisions and delegate when his own information is inconclusive either for or against adoption. Such selective use of delegation reduces manipulation by the defense secretary and increases the value of his expertise.

A recent literature stresses the benefits of delegation in terms of providing incentives for experts to gather information (Gilligan and Krehbiel 1987; Aghion and Tirole 1997; Dewatripont and Tirole 1999). In assuming that experts' private information is exogenously given, our delegation model focuses instead on the use of delegation mechanisms by decision makers to mitigate the agency problem of information manipulation. In ou model the decision to be made is binary. This mutes a main issue in the existing literature on delegation of how the decision maker should restrict the freedom of choice in delegating the decision to experts (Holmstrom 1984; Melumad and Shibano 1991; Armstrong 1994). We are able to isolate and examine how other aspects of delegation, including recruitment of experts and control of decision making by an expert team, contribute to balancing the need to minimize loss of control and the need to make use of expertise. The assumption of binary decisions in our setup also implies that the revelation of information is consistent only with delegation in the case of an uninformed 
decision maker and a single privately informed expert. This helps us steer away from the issue of how delegation (letting the expert decide) compares with communication (asking for the expert's recommendation) for the decision maker (Gilligan and Krehbiel 1987; Krishna and Morgan 2000; Dessein 2002). More generally, in our model the decision maker has no incentives to overrule the decision reached by a delegated expert team. Thus delegation mechanisms discussed in the present paper do not require excessive commitment ability on the part of the decision maker.

\section{A Binary Decision Model of Expertise}

The framework of our model is borrowed from optimal statistical decisions (DeGroot 1970). To facilitate the exposition, we adopt the language of policy evaluation. A policy change under consideration has either positive or negative net benefit. The decision to be made is either to "adopt" or "reject" the policy change. For convenience, we shall think of rejection of the policy change as the status quo choice.

There are two types of agents who may be involved in the decision making: decision maker $D$, who has the formal authority to make the decision, and experts $A$ and $B$, who may be delegated by $D$ to make the decision. We use superscript $d$ to represent the notation for the decision maker and superscripts $a$ and $b$ to represent the notation for experts $A$ and $B$, respectively. We specify the preferences of any agent $i=d, a$, $b$ as follows. Let $\gamma^{i}$ be the prior of the agent that the policy change has positive net benefit. Denote as $\lambda_{1}^{i}$ the utility cost of type I error (false adoption) and $\lambda_{2}^{i}$ the utility cost of type II error (false rejection). ${ }^{1}$ Each agent wishes to minimize expected cost. Write $k_{1}^{i}=\lambda_{1}^{i}\left(1-\gamma^{i}\right)$ and $k_{2}^{i}=\lambda_{2}^{i} \gamma^{i}$. The ratio $k^{i}=k_{1}^{i} / k_{2}^{i}$ represents the expected cost of false adoption relative to false rejection under the prior and completely characterizes the preferences of the agent. ${ }^{2} \mathrm{~A}$ greater $k^{i}$ means that the agent is more prone to rejection. We say that agent $i$ has "neutral" preferences if $k^{i}=1$. If $k^{i}<1$, we call agent $i$ a "liberal." If $k^{i}>1$, we call him a "conservative." Throughout the paper, we assume that preferences of all agents are common knowledge.

Information structure is modeled as follows. Each agent $i=d, a, b$, if informed, privately observes a one-dimensional signal $y^{i}$. The signal $y^{i}$ is assumed to be distributed on $[y, \bar{y}]$ (the bounds can be infinity), with differentiable density function $f_{p}^{i}$ and corresponding distribution

\footnotetext{
'The cost of making the contect decision (i.e. adopt when the policy change has positive benefit and reject when the policy has negative benefit) is normalized to zero.

"There is no difference in this model between bias as manifested in $\gamma$ ' and preferences as manifested in $\lambda_{1}$ and $\lambda_{i}^{\prime}$ only the matio $k$ maters. We use the words "bias" and "preferences" incerchangeably:
} 
function $F_{p}^{i}$ conditional on the state that the policy change has positive net benefit, and with $f_{n}^{i}$ and $F_{n}^{i}$ conditional on the state that the policy change has negative benefit. ${ }^{3}$ For simplicity, when multiple informed agents are involved, we assume that the signals are independent conditional on the true state.

We introduce some notation that will be used throughout the paper. For any signal $y^{i}$, let $l^{i}\left(y^{i}\right)$ denote the likelihood ratio $f_{p}^{i}\left(y^{i}\right) / f_{n}^{i}\left(y^{i}\right)$. Write $L_{*}^{i}\left(y^{i}\right)=F_{p}^{i}\left(y^{i}\right) / F_{n}^{i}\left(y^{i}\right)$ and $L_{* *}^{i}\left(y^{i}\right)=\left[1-F_{p}^{i}\left(y^{i}\right)\right] /\left[1-F_{n}^{i}\left(y^{i}\right)\right]$. For any $y_{2}^{i}>y_{1}^{i}$, denote as $\mathcal{L}^{i}\left(y_{2}^{i}, y_{1}^{i}\right)$ the ratio $\left[F_{p}^{i}\left(y_{2}^{i}\right)-F_{p}^{i}\left(y_{1}^{i}\right)\right] /\left[F_{n}^{i}\left(y_{2}^{i}\right)-F_{n}^{i}\left(y_{1}^{i}\right)\right]$. We assume that the monotone likelihood ratio property holds; that is, $l^{i}\left(y^{i}\right)$ is strictly increasing (Milgrom 1981). A higher observed value of $y^{i}$ is stronger evidence that the policy change has positive net benefit. It is easy to verify that a strict monotone likelihood ratio property implies the following facts.

FACT 1. $L_{*}^{i}\left(y^{i}\right)<1<L_{.}^{i} .\left(y^{i}\right)$ for any $y^{i} \in(y, \bar{y})$.

FACT 2. $L_{*}^{i}\left(y^{i}\right)<l^{i}\left(y^{i}\right)<L_{* *}^{i}\left(y^{i}\right)$ for any $y^{i} \in(y, \bar{y})$.

FACT 3. $L_{*}^{i}\left(y^{i}\right)$ and $L_{* *}^{i}\left(y^{i}\right)$ are both strictly increasing functions.

FACT 4. $\mathcal{L}^{i}\left(y_{2}^{i}, y_{1}^{i}\right)$ is strictly increasing in both arguments for all $y_{2}^{i}>$ $y_{1}^{i}$.

Finally, for the analysis with multiple informed agents, we also assume that $l^{i} / L_{*}^{i}$ and $l^{i} / L_{*}^{i}$. are increasing functions in order to carry out comparative statics exercises. These assumptions are satisfied by, for example, normal distributions with a shift in mean to represent the true state.

Decision maker $I$ may decide to dictate the decision on the basis of his own prior or information, without consulting any expert. Alternat tively, $D$ may decide to delegate the decision to an expert or a team of experts. We assume that in delegating the decision, the decision maker can commit to not overuling the decision by the experts. The choice between dictating the decision and delegating it may seem to be an artificial one, in the sense that in reality decision makers often have other means of eliciting the expert information, such as ashing the

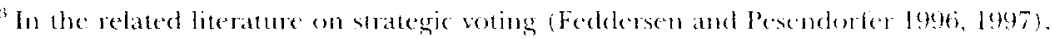
the signals as well as the states are binaly. Here we follow (rawford and Soldel (lose) in assuming continuously distributed signals. This allows un to study strategic infomation transmission and aggregation in tems of theshold strategies. which are more intuitive and less cumbersome than mixed strategies in models of binary signals. (Other papers

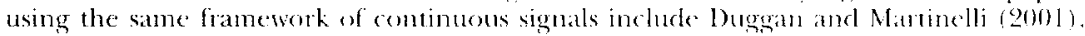
Li $(2001)$, and $\mathrm{Li}$, Rosen, and Suen $(2001)$

"This assumption is made to facilitate the presentation. We shall we below that comb mitment is not needer in the case of delegating the decinion to a single expert, either by an uninformed decision mater or by an informed one, because the willingness ws delegate is itself a credible commiment to not oxcombing the expert. In the case of delegating to a team of experts, the commitment to mot axcrubling the teames decision has value only when the decision maker monitors how the tean makes the decision after delegation.
} 
experts for a recommendation and promising to act on it in a particular wav. However, as a decentralized way of resolving the conflicts between the decision maker and the experts and making use of the expertise, clelegation has a simple administrative structure that makes it easy to implement. Further, for the decision maker, delegation is a convincing commitment to allowing the experts to have their say. As we shall see below in some contexts, the decision maker cannot do better than simply delegating the decision to the experts.

\section{Delegating Decisions to a Single Expert}

In this section, we assume that decision maker $D$ is uninformed. A liberal decision maker will choose adoption, and a conservative decision maker will choose rejection.

If decision maker $D$ delegates the decision to expert $A$, the expert will make the decision on the basis of the observed value of $y^{a}$. The expert uses Bayes' rule to update the posterior probabilities that the policy change has positive and negative net benefits to $\eta \gamma^{a} f_{p}^{a}\left(y^{a}\right)$ and $\eta\left(1-\gamma^{a}\right) f_{n}^{a}\left(y^{a}\right)$, respectively, where $\eta$ is a normalizing factor to make the probabilities sum to one. Expert $A$ will adopt if $\eta\left(1-\gamma^{a}\right) f_{n}^{a}\left(y^{a}\right) \lambda_{1}^{a} \leq$ $\eta \gamma^{a} f_{p}^{a}\left(y^{a}\right) \lambda_{2}^{a}$. This condition reduces to the rule that $A$ will choose adoption if $y^{a} \geq \hat{t}^{a}$ and choose rejection otherwise, where $\hat{t}^{a}$ is defined by $l^{a}\left(\hat{t}^{a}\right)=k^{a}$ (DeGroot 1970).

According to expert $A$ 's optimal decision rule, the expected cost $C^{d}$ to $D$ is

$$
C^{d}=k_{1}^{d}\left[1-F_{n}^{a}\left(\hat{t}^{a}\right)\right]+k_{2}^{d} F_{p}^{a}\left(\hat{t}^{a}\right) .
$$

Thus, if the decision maker is a liberal, he prefers delegating the decision to expert $A$ to making an uninformed adoption decision if and only if $k_{1}^{d} \geq C^{d}$. This is equivalent to the requirement that $k^{d} \geq L^{a}$. $\left(\hat{t}^{a}\right)$. Similarly, if the decision maker is a conservative, he prefers delegating the decision to expert $A$ to making an uninformed rejection decision if and only if $k_{2}^{d} \geq C^{d}$, which is equivalent to $k^{d} \leq L_{*}^{a} .\left(\hat{t}^{a}\right)$. Note that $L_{*}^{a}\left(\hat{t}^{a}\right)<1<$ $L_{*}^{a} .\left(\hat{t}^{a}\right)$ (fact 1$)$. We summarize these results in the following proposition.

Proposition 1 . Decision maker $D$ dictates adoption if he is sufficiently liberal $\left(k^{d}<L_{*}^{a},\left(\hat{t}^{a}\right)\right)$, dictates rejection if he is sufficiently conservative $\left(k^{d}>L_{* *}^{a}\left(\hat{t}^{a}\right)\right)$, and delegates the decision to expert $A$ if $k^{d}$ is in between these critical values.

In our discussion, we have assumed that the decision maker can commit to not overruling the expert after the expert has made the decision. It turns out that this commitment is indeed credible if $k^{d}$ is between $L_{*}^{a}\left(\hat{t}^{a}\right)$ and $L_{*}^{a} .\left(\hat{t}^{a}\right)$. To see this, suppose that expert $A$ has made a decision to adopt. The decision maker infers that $y^{a} \geq \hat{t}^{a}$. Decision maker $D$ there- 
fore updates his posterior probabilities that the policy change has positive and negative net benefits to $\eta \gamma^{d}\left[1-F_{p}^{a}\left(\hat{t}^{a}\right)\right]$ and $\eta\left(1-\gamma^{d}\right)[1-$ $\left.F_{n}^{a}\left(\hat{t}^{a}\right)\right]$, respectively (where $\eta$ is a normalizing factor to make the probabilities sum to one). On the basis of these updated probabilities, the decision maker would choose adoption over rejection if $k^{d} \leq L_{* *}^{a}\left(\hat{t}^{a}\right)$, implying that it would not be optimal to overrule the expert's adoption decision. Similar reasoning suggests that $D$ has no incentive to overrule A's rejection decision since $k^{d} \geq L_{*}^{a}\left(\hat{t}^{a}\right)$. Thus, provided that the decision maker finds that delegating the decision to the expert is better than making the decision by himself, he has no incentive to overrule the expert subsequent to learning the expert's decision.

More generally, we can show that the decision maker cannot do better than delegation by committing to any mechanism to elicit the information from the experts. Since the decision maker makes a binary choice even though the underlying signal is continuous, standard arguments imply that in any incentive-compatible mechanism, the probability of adoption can take at most two values. The coarsening of information in incentive schemes is a generic feature of strategic information transmission (Crawford and Sobel 1982). ${ }^{5}$ Furthermore, the best that an incentive-compatible mechanism can achieve for the decision maker is to choose adoption if $y^{a} \geq \hat{t}^{a}$ and rejection if $y^{a}<\hat{t}^{a}$, which is equivalent to delegating the decision to the expert. Thus delegating to the expert corresponds to the best possible mechanism.

\section{A. Advocacy}

The range $\left[L_{*}^{a}\left(\hat{t}^{a}\right), L_{* *}^{a}\left(\hat{t}^{a}\right)\right]$ represents the range of preferences such that any decision maker with $k^{d}$ in this range will delegate the decision to $A$. Since $L_{*}^{a}\left(\hat{t}^{a}\right)<1<L_{* *}^{a}\left(\hat{t}^{a}\right)$ for any $k^{a}$, a neutral decision maker $\left(k^{d}=1\right)$ is willing to delegate decisions to an informed expert with any bias $k^{a}$. Further, since $L_{*}^{a}\left(y^{a}\right)<l^{a}\left(y^{a}\right)<L_{* *}^{a}\left(y^{a}\right)$ for any $y^{a}$ (fact 2), substituting $y^{a}=\hat{t}^{a}$ into this inequality yields $L_{*}^{a}\left(\hat{t}^{a}\right)<k^{a}<L_{* *}^{a}\left(\hat{t}^{a}\right)$. A decision maker is willing to delegate decisions to an expert if $k^{d}=k^{a}$. More generally, if the expert is a liberal, any decision maker who is a less extreme liberal $\left(k^{d} \in\left[k^{a}, 1\right]\right)$ will find this expert's service useful. If the expert is a conservative, any decision maker who is a less extreme conservative $\left(k^{d} \in\left[1, k^{a}\right]\right)$ will find such an expert useful. However, even a greater bias than the expert's, or an opposite bias, does not rule out delegation by the decision maker. As long as the decision maker is not extremely biased, he is willing to delegate decisions to make use of the expert's information.

\footnotetext{
${ }^{5}$ See also Green and Stokey (1980). Gilligan and Krehbiel (1989) and Austen-Smith (1990) apply the signaling model to agenda-setting in legislatures.
} 
Since $d \hat{t}^{a} / d k^{a}>0$ and since $L_{*}^{a}$ and $L_{* *}^{a}$ are strictly increasing functions (fact 3 ), the boundaries of the delegation range, $L_{*}^{a}\left(\hat{t}^{a}\right)$ and $L_{* *}^{a}\left(\hat{t}^{a}\right)$, are increasing functions of $k^{a}$. Thus an expert with a more extreme preference for rejection has a greater chance of being delegated the decision by decision makers who are conservatives. Intuitively, for a decision maker who is a conservative, the uninformed decision is to reject. Delegating the decision to expert $A$ makes a difference to the decision maker only if expert $A$ decides to adopt. When the expert is more biased toward rejection, a decision to adopt requires more overwhelming evidence that the policy change will have positive net benefit, which implies that more conservative decision makers will find delegation to $A$ acceptable.

Of course an increase in $k^{a}$ raises the lower boundary of delegation $L_{*}^{a}\left(\hat{t}^{a}\right)$ as well as the upper boundary $L_{* *}^{a}\left(\hat{t}^{a}\right)$. Although an expert with a greater bias for rejection is acceptable to a wider spectrum of conservative decision makers, fewer liberal decision makers will agree to delegate the decision to this expert. This poses a problem for an organization trying to recruit experts to ensure their wide use, because decision makers biased in opposite directions cannot be accommodated at the same time.

Another way of thinking about the delegation range is to consider the range of experts who will be useful to a decision maker with a given preference parameter $k^{d}$. A liberal decision maker $\left(k^{d}<1\right)$ will not benefit from the advice of an expert whose preferences are sufficiently to the right, and vice versa for a conservative decision maker $\left(k^{l}>1\right)$. This is consistent with the observation that people have a tendency to rely on information from like-minded sources. According to our theory, a politically conservative decision maker listens to the Heritage Foundation (a conservative think tank) not because he enjoys voices that agree with his own political inclinations, but because his decisions will be changed if the Heritage Foundation recommends a policy that is contrary to his preferences. Similarly, this politically conservative decision maker does not read the Nation (a liberal publication), not because he dislikes reading dissenting opinion, but because whatever policy recommendations that emerge from this magazine will not change his prior sufficiently to alter his decisions. Our model offers a decision-theoretic explanation rather than a taste-based explanation for the demand for biased opinion. ${ }^{6}$

\footnotetext{
"That the value of information lies in its potential to change a decision has been pointed out by, among others, Calvert (1985) and Meyer (1991). In different contexts, both authors demonstrate the value of introducing bias into the structure of information when information is coarse.
} 


\section{B. Value versus Influence}

We distinguish between two aspects that make an expert useful in an organization: value and influence. An expert is "influential" if his service has positive value to a wide range of decision makers in the organization. The extent of influence of an expert $A$ is measured by the delegation range $\left[L_{*}^{a}\left(\hat{t}^{a}\right), L_{* *}^{a}\left(\hat{t}^{a}\right)\right]$ described above. Another aspect of expertise is the value of his service to a decision maker with given preferences. Conditional on his delegating the decision to expert $A$, the expected $\operatorname{cost} C^{d}$ in equation (1) measures the benefit from delegation to decision maker $D$. Taking derivatives and using the definition of $\hat{t}^{a}$, we find that $d C^{d} / d k^{a}$ has the same sign as $\left(k^{a}-k^{d}\right) d \hat{t}^{a} / d k^{a}$. Since $d \hat{t}^{a} / d k^{a}>0$, the expected cost $C^{d}$ increases in $k^{a}$ if and only if $k^{a}>k^{d}$, and $C^{d}$ decreases in $k^{a}$ if and only if $k^{a}<k^{d}$. In other words, the value of an expert's service to a decision maker falls as the expert's preferences diverge from the decision maker's.

An expert is useless if decision makers do not want to delegate their decisions to him. When recruiting an expert, an organization may want to ensure that a wide range of decision makers with different preferences will want to use the expert's service. However, there is a trade-off between recruiting an expert with wide influence and recruiting one with great value. Suppose that the likely decision makers in the organization are liberals $\left(k^{d}<1\right)$. To ensure that the expert within this organization is influential, the organization should pick an expert whose preferences are strongly biased toward adoption. But such choice also means that the expert has small value to any given decision maker in the organization, because very often the strongly biased expert will simply reinforce the tendency of the decision makers to adopt. ${ }^{7}$

\section{Delegating Decisions to a Team of Experts}

When decisions are delegated to a single expert, this expert decides according to his own preferences, which do not necessarily accord with those of the decision maker. One way to control manipulation by a single expert is to play one expert against another. In other words, conflicts of interest among the experts themselves may prevent the decision maker from being dominated by any single expert. An additional advantage from using more than one expert arises from potential gains

\footnotetext{
${ }^{7}$ Another factor that affects the value of an expert is the quality of his information. In our setup, we can model quality of information by considering a modification of the information structure available to expert $A$. Suppose that the expert observes his private signal with probability $1-\pi^{n}$ and observes the true state with probability $\pi^{\prime \prime}$. An increase in $\pi$ "represents a higher quality of $A$ 's information. We can show that this leads to both a higher value from delegation for any given decision maker and a wider range of delegation.
} 
from pooling diverse information. We illustrate these issues in the simplest terms by considering a team of two experts.

An uninformed decision maker $D$ considers whether to dictate the outcome or to delegate to a team of two experts, $A$ and $B$. If delegation occurs, experts $A$ and $B$ face a problem of strategic information aggregation. Such a game is analyzed by Li et al. (2001). For the purposes of the present paper, we focus on simple two-way voting equilibria, corresponding to types of two-way voting procedures. In "unilateral adoption," the policy change is adopted if there is at least one "adopt" vote. In "unilateral rejection," adoption requires two "adopt" votes. These two voting procedures have natural interpretations as two different "delegation mechanisms." In contrast to the case of a single expert, in delegation to a team of experts, it is no longer self-evident how the decision maker lets the experts have their say. Some degree of control and monitoring is necessary. The unilateral adoption and unilateral rejection procedures impose minimum structure on the game of strategic information aggregation between the two experts. They are easy to implement in practice and provide a tractable framework for our analysis of delegation and decision maker-expert relationships.

The two delegation mechanisms define two different voting games between $A$ and $B$. Suppose that there exists a pair of thresholds $\left(t_{*}^{a}, t_{*}^{b}\right)$ that satisfy

$$
\begin{aligned}
& l^{a}\left(t_{*}^{a}\right) L_{*}^{b}\left(t_{*}^{b}\right)=k^{a}, \\
& l^{b}\left(t_{*}^{b}\right) L_{*}^{a}\left(t_{*}^{a}\right)=k^{b} .
\end{aligned}
$$

Similarly, suppose that a pair of thresholds $\left(t_{* *}^{a}, t_{* *}^{b}\right)$ satisfy

$$
\begin{aligned}
& l^{a}\left(t_{* *}^{a}\right) L_{* *}^{b}\left(t_{* *}^{b}\right)=k^{a}, \\
& l^{b}\left(t_{* *}^{b}\right) L_{* *}^{a}\left(t_{* *}^{a}\right)=k^{b} .
\end{aligned}
$$

We use the following result by Li et al. (2001).

Proposition 2. In the voting game with unilateral adoption, each expert $e=a, b$ votes "adopt" if and only if $y^{e} \geq t_{*}^{e}$. In the voting game with unilateral rejection, each expert $e=a, b$ votes "adopt" if and only if $y^{e} \geq t_{* *}^{e}$.

Boundary conditions on $l^{e}$ and $L^{e}$ can be made to ensure the existence of the equilibrium thresholds ( $\mathrm{Li}$ et al. 2001). Under the assumption that $l \% L_{*}^{e}$ and $l \% L_{* *}^{e}$ are increasing functions for $e=a, b$, each of the equilibria described above is unique. 


\section{A. Balance}

Let $C_{*}^{d}$ be the expected cost to decision maker $D$ of delegating his decision to a team of experts $A$ and $B$ under unilateral adoption. We have

$$
C_{*}^{d}=k_{1}^{d}\left[1-F_{n}^{a}\left(t_{*}^{a}\right) F_{n}^{b}\left(y_{*}^{b}\right)\right]+k_{2}^{d} F_{p}^{a}\left(t_{*}^{a}\right) F_{p}^{b}\left(t_{*}^{b}\right) .
$$

Suppose that the decision maker is a liberal. He prefers delegating the decision to this team to making an uninformed adoption if $k_{1}^{d} \geq C_{*}^{d}$. This is equivalent to the condition that $k^{d}$ be greater than the critical value $L_{*}^{a}\left(t_{*}^{a}\right) L_{*}^{b}\left(t_{*}^{b}\right)$.

Similarly, let $C_{* *}^{d}$ be the expected cost under the unilateral rejection delegation mechanism. We have

$$
\begin{aligned}
C_{* *}^{d}= & k_{1}^{d}\left[1-F_{n}^{a}\left(t_{* *}^{a}\right)\right]\left[1-F_{n}^{b}\left(t_{* *}^{b}\right)\right] \\
& +k_{2}^{d}\left\{1-\left[1-F_{p}^{a}\left(t_{* *}^{a}\right)\right]\left[1-F_{p}^{b}\left(t_{* *}^{b}\right)\right]\right\} .
\end{aligned}
$$

When the decision maker is a conservative, he prefers delegating the decision to this team to making an uninformed rejection decision if $k_{2}^{d} \geq C_{* *}^{d}$. This is equivalent to requiring that $k^{d}$ be smaller than the critical value $L_{* *}^{a}\left(t_{* *}^{a}\right) L_{* *}^{b}\left(t_{* *}^{b}\right)$. Note that

$$
L_{*}^{a}\left(t_{*}^{a}\right) L_{*}^{b}\left(t_{*}^{b}\right)<1<L_{* *}^{a}\left(t_{* *}^{a}\right) L_{* *}^{b}\left(t_{* *}^{b}\right)
$$

(fact 1). We summarize the results of the discussion in the following proposition. $^{8}$

Proposition 3. Decision maker $D$ prefers an uninformed adoption decision to delegation under unilateral adoption if he is sufficiently liberal $\left(k^{d}<L_{*}^{a}\left(t_{*}^{a}\right) L_{*}^{b}\left(t_{*}^{b}\right)\right)$, prefers an uninformed rejection decision to delegation with unilateral rejection if he is sufficiently conservative $\left(k^{d}>L_{* *}^{a}\left(t_{* *}^{a}\right) L_{* *}^{b}\left(t_{* *}^{b}\right)\right)$, and prefers delegation if $k^{d}$ is in between these critical values.

In deriving proposition 3, we have assumed that the decision maker can credibly commit to not overruling the decision by the team after delegation. It turns out that if the decision maker is willing to delegate the decision under a given mechanism, then he will not have incentives to overrule the team's decision when he does not monitor how the team reaches the decision. For example, suppose that the decision maker

\footnotetext{
${ }^{8}$ Although making an uninformed decision is dominated by delegation for decision maker $D$ when $k^{d} \in\left[L_{*}^{a}\left(t_{*}^{a}\right) L_{*}^{b}\left(t_{*}^{b}\right), L_{*}^{a} .\left(t_{*}^{a}\right) L_{*}^{b}\left(t_{*}^{b}\right)\right]$, proposition 3 does not directly compare $C_{*}^{d}$ and $C_{*}^{d}$. Such comparisons would depend on the distribution functions of the data. With arbitrary distributions satisfying the monotone likelihood ratio property, we cannot rule out the possibility that decision maker $D$ prefers delegation under unilateral rejection to making an informed adoption decision when $k^{d}<L_{*}^{a}\left(t_{*}^{a}\right) L_{*}^{b}\left(t_{*}^{b}\right)$. Similarly, we cannot prove that decision maker $D$ will not prefer delegation under unilateral adoption to making an informed rejection decision when $k^{d}>L_{*}^{a} .\left(t_{*}^{a}\right) L_{*}^{b} .\left(t_{*}^{b}\right)$. In light of these observations, the range of delegation should be viewed as a conservative bound.
} 
agrees to delegate the decision under unilateral adoption. Then, after learning that the team has made the rejection decision, $D$ infers that $y^{a}<t_{*}^{a}$ and $y^{b}<t_{*}^{b}$. The decision maker has no incentive to overrule this decision because overruling requires that $k^{d}<L_{*}^{a}\left(t_{*}^{a}\right) L_{*}^{b}\left(t_{*}^{b}\right)$, in which case $D$ would not have agreed to delegate in the first place. Similarly, after learning that the team has made the adoption decision, $D$ infers that $y^{a} \geq t_{*}^{a}$ or $y^{b} \geq t_{*}^{b}$, or both. Overruling this decision requires $k^{d}>[1-$ $\left.F_{g}^{a}\left(t_{*}^{a}\right) F_{g}^{b}\left(t_{*}^{b}\right)\right] /\left[1-F_{n}^{a}\left(t_{*}^{a}\right) F_{n}^{b}\left(t_{*}^{b}\right)\right]$, but the opposite is true because $D$ prefers delegation to making an uninformed rejection decision. Therefore, as in the case of a single expert, for a given delegation mechanism the very willingness of $D$ to delegate the decision is itself a credible commitment to not overruling the team's decision. ${ }^{9}$ Moreover, the preceding analysis implies that if the procedure itself is optimally chosen, the decision maker has no incentive to overrule the team's decision if $k^{d}$ falls into the delegation range $\left[L_{*}^{a}\left(t_{*}^{a}\right) L_{*}^{b}\left(t_{*}^{b}\right), L_{* *}^{a}\left(t_{* *}^{a}\right) L_{* *}^{b}\left(t_{* *}^{b}\right)\right]$.

Several corollaries are immediate from proposition 3 . First, since $L_{*}^{a}\left(t_{*}^{a}\right) L_{*}^{b}\left(t_{*}^{b}\right)<1<L_{* *}^{a}\left(t_{* *}^{a}\right) L_{* *}^{b}\left(t_{* *}^{b}\right)$ for any $k^{a}$ and $k^{b}$, a neutral decision maker will always prefer delegation to making an uninformed decision. Second, since $L_{*}^{a}\left(t_{*}^{a}\right) L_{*}^{b}\left(t_{*}^{b}\right)<k^{a}, k^{b}$ and $L_{* *}^{a}\left(t_{* *}^{a}\right) L_{* *}^{b}\left(t_{* *}^{b}\right)>k^{a}, k^{b}$, any decision maker with preferences $k^{d}$ between $k^{a}$ and $k^{b}$ will always prefer delegating the decision to the team of experts $A$ and $B$ to making the decision himself. Third, since a team of two experts has access to more information than a single expert, one expects that the delegation range is larger with two experts than with one expert. To verify this claim, note that from $l^{a}\left(\hat{t}^{a}\right)=k^{a}$ and $l^{a}\left(t_{*}^{a}\right) L_{*}^{b}\left(t_{*}^{b}\right)=k^{a}$, we have $t_{*}^{a}>\hat{t}^{a}$. Since $L_{*}^{b}\left(t_{*}^{b}\right)=k^{a} / l^{a}\left(t_{*}^{a}\right)$, the lower bound of the delegation range for an expert team is $k^{a} L_{*}\left(t_{*}^{a}\right) / l\left(t_{*}^{a}\right)$. In contrast, the lower bound of the delegation range for the single expert $A$ is $k^{a} L_{*}\left(\hat{t}^{a}\right) / l\left(\hat{t}^{a}\right)$. Since $t_{*}^{a}>\hat{t}_{a}$, the assumption that $l^{a} / L_{*}^{a}$ is a strictly increasing function implies that the lower bound for the team is smaller than for the single expert. A similar argument establishes that the upper bound for the team is higher than for the single expert $A$. Thus a team of experts $A$ and $B$ is acceptable to a wider range of decision makers than either of them alone is. From the decision maker's point of view, adding a second expert $B$ cannot reduce his willingness to delegate the decision. In the worst cases, when $k^{b}$ is close to either zero or infinity, the extreme expert can be rendered irrelevant by an appropriate choice of the delegation mechanism. For example,

\footnotetext{
"The resemblance between delegating to two experts and delegating to a single expert in terms of commitment to not overruling the decision breaks down if $D$ monitors how the team makes the decision. For example, suppose that $k^{u}=k^{b}$ and the two signals have the same conditional distributions. We can easily verify that there is an interval of $k^{t}$ such that $D$ will agree to delegate under unilateral adoption but will have incentives to overrule if the team's adoption decision is unilateral, though not if the team reaches either adoption or rejection decisions unanimously.
} 
if expert $B$ is an extreme liberal ( $k^{b}$ close to zero), the decision maker can choose unilateral rejection so that delegating the decision to the team of experts $A$ and $B$ is equivalent to delegating to just $A$. Finally, under either unilateral adoption or unilateral rejection, as $k^{b}$ increases, expert $B$ raises his threshold of adoption and expert $A$ lowers his in response. This can be established by taking total derivatives of equations (2) and using the assumption that $l^{e} / L_{*}^{e}(e=a, b)$ is an increasing function. Then one can show that the overall effect of an increase in $k^{b}$ on the range of delegation is unambiguous: for any fixed $k^{a}$, as $k^{b}$ increases, both the lower bound and the upper bound of the delegation range $\left[L_{*}^{a}\left(t_{*}^{a}\right) L_{*}^{b}\left(t_{*}^{b}\right), L_{* *}^{a}\left(t_{* *}^{a}\right) L_{* *}^{b}\left(t_{* *}^{b}\right)\right]$ rise. Thus a team with an expert who has more extreme preferences for rejection is more widely acceptable among decision makers who are conservatives but is less acceptable among decision makers who are liberals.

We have seen in the previous section that decision makers biased in opposite directions cannot be accommodated at the same time by any single expert. This problem can be avoided with two experts. An organization can ensure a wide use of delegation by different types of decision makers if the team of two experts have extreme and opposite preferences. Such a balanced team of radicals will have a wider delegation range than a one-sided team of moderates. To see this, consider a team of two experts $A$ and $B$ with moderate preferences $k^{a}$ and $k^{b}$, with $k^{a}<k^{b}$. Let $\left[L_{*}^{a}\left(t_{*}^{a}\right) L_{*}^{b}\left(t_{*}^{b}\right), L_{* *}^{a}\left(t_{* *}^{a}\right) L_{* *}^{b}\left(t_{* *}^{b}\right)\right]$ be the corresponding delegation range. Any decision maker with preference $k^{d}$ sufficiently biased toward rejection $\left(k^{d}>L_{* *}^{a}\left(t_{* *}^{a}\right) L_{* *}^{b}\left(t_{* *}^{b}\right)\right)$ prefers making an uninformed decision to delegating the decision to this team. This problem can be overcome by recruiting another expert with a higher $k^{b}$. However, doing so without changing $k^{a}$ will make the team less acceptable to decision makers who are liberals. The solution is to reduce $k^{a}$ while increasing $k^{b}$, that is, recruit experts who are more extreme in opposite directions. Consider another team of two experts $A_{1}$ and $B_{1}$ with extreme and opposite preferences, say $k^{a_{1}}<L_{*}^{a}\left(t_{*}^{a}\right) L_{*}^{b}\left(t_{*}^{b}\right)$ and $k^{b_{1}}>L_{* *}^{a}\left(t_{* *}^{a}\right) L_{* *}^{b}\left(t_{* *}^{b}\right)$. Then, since any decision maker with preferences $k^{d} \in\left[k^{a_{1}}, k^{b_{1}}\right]$ is willing to delegate the decision to this other team, the range of delegation for the second team with experts $A_{1}$ and $B_{1}$ strictly contains the range of delegation for the first team with experts $A$ and $B$.

A balanced team has a greater use of delegation than a one-sided team because the decision maker can specify the voting procedure (unilateral adoption or unilateral rejection) in delegating to a team of experts. When the decision maker is a liberal, his natural ally is the expert who has the same bias relative to the other expert, and the delegation mechanism of unilateral adoption protects the interests of the decision maker by giving the control of decision making in the team to his ally. Similarly, when the decision maker is a conservative, he relies morc on 
the expert who is relatively biased toward rejection, and the procedure of unilateral rejection protects the interests of the decision maker.

The claim has often been made that "competition of ideas" by advocates with opposing interests is the best way to bring out the true merits of contrasting views in a debate. The claim has been cited as one advantage of the adversarial judicial system of the Anglo-American kind over the inquisitorial system found in continental Europe. In Dewatripont and Tirole (1999), it is cheaper to provide incentives for two competing advocates to gather information than for a single expert who is responsible for collecting evidence for both sides of the debate. Their argument does not hinge on eliciting private information from experts; indeed, the advantage of an adversarial system may be reduced if the collected evidence is private. In our model the benefit of having balanced interests on the expert team does not come from forcing experts with opposing interests to compete with each other. The issue of balance in an expert team has recently also been studied by Krishna and Morgan (2001). As in the present paper, their model of expertise deals with two experts on a team who have different preferences regarding the decision; but in contrast to our model, these experts have the same data. The authors show that there is benefit for a decision maker to consult both experts (instead of consulting the one whose preference is closer to that of the decision maker) only when they are biased in opposite directions relative to the decision maker. The apparent benefit from balancing the expert team with opposite biases arises because eliciting private information is easier for the decision maker when the two experts are opposed in their interests. In our model, balancing the expert team makes the delegation more likely for a wider range of biases of the decision maker, because the decision maker can always adopt the appropriate delegation mechanism to rely on the more "loyal" expert.

However, using a balanced team of experts with extreme preferences is not costless. Such a team may be very influential in the sense that it is acceptable to a wide range of decision makers, but the value of its expertise, while positive, is generally low. Greater conflict of interest within a balanced team of extremists makes information transmission less efficient. As a result the quality of the team's decision deteriorates. To see this point, consider the expected cost for decision maker $D$ if he uses a team of experts $A$ and $B$. Suppose $k^{a}<k^{d}<k^{b}$. Under unilateral adoption, this expected cost is given by equation (4) above. If a balanced but more extreme team of experts (with $k^{a_{1}}<k^{a}$ and $k^{b_{1}}>k^{b}$ ) is used, then the adoption threshold $t_{*}^{a}$ falls and $t_{*}^{b}$ rises. Since $\partial C_{*}^{d} / \partial t_{*}^{a}$ has the

\footnotetext{
"Milgrom and Roberts (1986) (see also Shin 1994) study the situation in which experts' information can be concealed but cannot be distorted. Another recent paper on the problem of multiple experts is Ottaviani and Sorensen (1999); in their model the experts are concerned with making recommendations that are validated ex post.
} 
same sign as $k^{a}-k^{d}$ and $\partial C_{*}^{d} / \partial t_{*}^{b}$ has the same sign as $k^{b}-k^{d}$, the expected cost $C_{*}^{d}$ for decision maker $D$ unambiguously rises. The same reasoning shows that the expected cost for decision maker $D$ under unilateral rejection $C_{* *}^{d}$ also rises when the team of experts becomes more biased in opposite directions. Thus there is again a trade-off between influence and value for an expert team. A team that is acceptable to a wide class of decision makers may not produce decisions that are highly valuable.

\section{B. Neutrality}

For a given decision maker $D$, the ideal team of experts should have both experts with preferences identical to D's. However, the analysis above shows that having balanced preferences in the expert team makes it widely acceptable to different decision makers. Thus there is a benefit of having some degree of conflict in the team. Consider, then, a situation in which decision maker $D$ already has expert $A$ at hand and is thinking about bringing another expert, say $B$, to the team. Suppose that $k^{\prime \prime}<$ $k^{\prime}$. Does decision maker $D$ want the second expert $B$ to have the same preference that he has $\left(k^{b}=k^{d}\right)$ ?

The answer to this question is no. To see this, decompose $d C_{*}^{d} / d k^{b}$ into two parts:

$$
\frac{d C_{*}^{d}}{d k^{b}}=\frac{\partial C_{*}^{d}}{\partial t_{*}^{a}} \frac{d t_{*}^{a}}{d k^{b}}+\frac{\partial C_{*}^{d}}{\partial t_{*}^{b}} \frac{d t_{*}^{b}}{d k^{b}} .
$$

The second term of equation (6) is zero at $k^{b}=k^{d}$ because $\partial C_{*}^{d} / \partial t_{*}^{b}$ has the same sign as $k^{b}-k^{d}$. Since $k^{d}<k^{a}$ and $d t_{*}^{a} / d k^{b}<0$, we get $d C^{d} / d k^{b}<0$. Thus decision maker $D$ does not want the new expert $B$ to have a preference $k^{b}$ identical to his. The reason is that doing so will force the existing expert $A$ to be more extreme and therefore distort $A$ 's information. Conflict of interest exists between experts within the team, as well as between expert $B$ and the decision maker. Since the cost of having an expert $B$ just slightly less biased (toward adoption) than he is has second-order importance, the decision maker will want the second expert to have the appearance of neutrality. This reduces $t_{*}^{a}$ and makes expert $A$ less manipulative with his information.

With some additional assumptions it can be shown that the best expert $B$ for the decision maker to recruit is one with $k^{b} \in\left(k^{d}, k^{a}\right)$. We make the assumption that the data of $A$ and $B$ have the same conditional distributions; this ensures that the effect of $B$ 's preferences $k^{b}$ on his threshold $t_{*}^{b}$ is greater in magnitude than the effect of $k^{b}$ on $A^{\prime}$ 's threshold $t_{*}^{a}$. We also assume that $k^{d}>L_{*}^{a}\left(t_{*}^{a}\right) L_{*}^{b}\left(t_{*}^{b}\right)$. This assumption is innocuous since decision maker $D$ would rather make an uninformed adoption 
decision if this condition is not satisfied. (The proof of proposition 4 is in the Appendix.)

Proposition 4. Suppose $k^{d}<k^{a}$. (i) For $k^{b} \leq k^{d}$, expected cost $C_{*}^{d}$ for the decision maker increases as $k^{b}$ falls. (ii) For $k^{b} \geq k^{a}, C_{*}^{d}$ increases as $k^{b}$ rises if experts $A$ and $B$ have the same conditional signal distributions and $k^{d} \geq L_{*}^{a}\left(t_{*}^{a}\right) L_{*}^{b}\left(t_{*}^{b}\right)$.

A similar argument leads to the conclusion that $d C_{* *}^{d} / d k^{b}<0$ for all $k^{b} \leq k^{d}$ and $d C_{* *}^{d} / d k^{b}>0$ for all $k^{b} \geq k^{a}$ when $k^{d} \leq L_{* *}^{a}\left(t_{* *}^{a}\right) L_{* *}^{b}\left(t_{* *}^{b}\right)$. Thus, regardless of whether the delegation mechanism is unilateral adoption or unilateral rejection, as long as the decision maker prefers delegation to making uninformed decisions, expected cost is minimized by choosing $k^{b}$ somewhere between $k^{d}$ and $k^{a}$.

The logic of proposition 4 does not depend on whether the decision maker is informed or not. In some situations a decision maker may sit on the committee himself or delegate an expert to sit on it. Proposition 4 suggests that even if the decision maker is equally well informed as the expert, he has an incentive to delegate the expert to sit on the committee on his behalf in order to reduce the conflicts of interest within the committee. By appointing someone with preferences more similar to those of other committee members, the decision maker commits himself to being less partisan and thus improves the pooling of information within the committee.

Proposition 4 offers a theory of neutrality in collective decision making. This theory is driven both by conflicts in interest between the decision maker and the experts and by the strategic manipulations of the two self-interested experts in the team. While we have illustrated this point under the assumption that the two experts play a simple two-way voting game, the same conclusion holds when more sophisticated mechanisms are considered. " For example, a decision maker or his delegate may make a recommendation, and a higher authority ultimately determines the outcome on the basis of his own private information and the recommendation. ${ }^{12}$ In this case, it is possible for the decision maker or his delegate to express different degrees of support for the proposal instead of just a "yes" or "no" recommendation. A different decision rule can be used depending on the recommendation. As long as conflict of interest exists between the decision maker and the higher authority, our theory suggests that the decision maker will recruit a delegate whose preferences are closer to those of the higher authority. This ensures that the higher authority will be less skeptical of the recommendation and therefore rely less on his own preferences and more on the rec-

\footnotetext{
${ }^{11}$ For a characterization of outcomes of these mechanisms, see Li et al. (2001).

${ }^{12}$ Letterie and Swank (1997) and Letterie, Swank, and van Dalen (2000) analyze the choicc of policy advisors in related contexts and arrive at conclusions similar to ours.
} 
ommendation in making the final decision. The decision maker benefits from the resulting improvement in the efficiency of information aggregation."

\section{Are Two Experts Betler than One?}

In concluding that the best expert for decision maker $l$ ) to recruit is one with preferences between those of the decision maker and those of the existing expert $A$, we have assumed that $I$ cannot exclude $A$ in delegating the decision. Is it possible that the conflicts between $D$ and $A$ are so large that $D$ wants to recruit an expert $B$ and delegate to $B$ alone?

If the delegation mechanism cannot be chosen, then having two experts can be worse than having just one for the decision maker. For example, a liberal decision maker prefers delegating to an expert with moderate preferences than to a team consisting of this expert and a hard-nosed conservative, if the team has to use the unilateral rejection mechanism. The reason is that the unilateral rejection mechanism allows the hard-nosed conservative member of the team to make the rejection decision by himself with little input from the moderate member.

Proposition 4 can be used to argue that this is not the case, as long as the decision maker can select the voting procedure in delegating the decision to the team of $A$ and $B$. To see this, suppose that $k^{d} \leq k^{b}$ and vary the preferences of expert $A$. If $k^{a}=0$, then under unilateral rejection, the team with $A$ and $B$ makes the same decisions as $B$ alone, because $A$ will always choose to adopt. Since $d C_{*}^{d} / d k^{a}<0$ for $k^{a} \leq k^{d}, D$ prefers the team to $B$ alone for all $k^{a} \leq k^{d}$. That is, from the decision maker's point of view, keeping a liberal expert $A$ on board is better as long as the delegation mechanism is unilateral rejection. On the other hand, if $k^{a}$ is arbitrarily large, then under unilateral adoption, the team makes the same decisions as $B$ alone. Under the same assumptions as in proposition 4 , we have $d C_{*}^{d} / d k^{a}>0$ for any $k^{a} \geq k^{b}$, which implies that

\footnotetext{
${ }^{1: 3}$ Inefficient information aggregation arises for two reasons: combinuous information is coarsened into discrete recommendations, and the thresholds for making different recommendations are chosen strategically. The result in proposition 4 implies that recruiting a delegate with preferenees closer to those of the higher decision maker would reduce the inefliciency from stratcgic choice of thresholds. An additional gain not related to proposition 4 is that lesser conflicts of interest between the cxpert and the higher decision maker would allow the expert to make finer gradations in his recommendation and hence reduce the incficiency from information coarsening.
} 
under unilateral adoption the team is better than $B$ alone. ${ }^{14}$ Thus keeping a conservative expert $A$ in the team is better as long as unilateral adoption is the procedure. Finally, if $k^{d}<k^{a}<k^{b}$, then the preceding argument implies that the team is better than $A$ alone; but since $A$ alone is better than $B$ alone when $k^{a}<k^{b}$, the team is better than $B$ alone. Thus, under the same assumptions as in proposition $4, D$ will never find it optimal to exclude $A$ in delegating the decision, regardless of how much $A$ 's preferences differ from his own. In combination with our earlier analysis, we have therefore established that a team of two experts has not only a greater influence than either of the two experts alone but also a greater value to any given decision maker.

\section{Informed Delegation}

So far we have assumed that the decision maker is uninformed, and the delegation decision can be based only on the extent of conflicts of interest between himself and the experts. In practice, decision makers often have their own source of information about the decision and seek expert advice only when needed. In this section, we consider delegation by a privately informed decision maker to a single expert. The delegation decision can be conditioned on the decision maker's information as well as on the preferences of the decision maker and the expert.

If the decision maker's information were known to the expert, informed delegation would be no different from uninformed delegation considered in the previous sections: we need only to change the decision maker's prior to the posterior given his information, and all the analysis follows through. The assumption that the decision maker's information is private means that the very act of delegating the decision communicates his information to the expert. By using selective delegation mechanisms, the decision maker can reduce manipulation by the expert in the event of delegation.

\footnotetext{
"A mumerical example shows that if the assumptions are not satisfied, using $B$ alone is better. Suppose that $k^{\prime \prime}=1.65$ and $k^{\prime \prime}=2.72$. For $B$, the conditional distributions are $N(0,1)$ and $N(1,1)$. If $D$ delegates to $B$ alone, we have $\hat{t}^{b}=1.5$ and $C^{d}=0.80$. Suppose that $k^{\prime \prime}=2.72$, but the conditional distributions of $A$ 's signal are $N(0,1)$ and $N(0.1,1)$ so that $A$ 's signal is much less informative than $B$ 's. When they form a team, unilateral adoption is better than unilateral rejection for 1 . But even under unilateral adoption, we have $l_{*}^{\prime \prime}=1.34, l_{*}^{\prime \prime}=1.52$, and $C_{*}^{\prime \prime}=0.87>C^{\prime \prime}$. The intuition is that under unilateral adoption, $B$ votes to adopt more cautiously than when he alone is delegated the decision (i.e., $\left.I_{*}^{\prime \prime}>\hat{t}^{\prime}\right)$. From the liberal decision maker's point of view, teaming $B$ with $A$ makes $B$ too conservative. This adverse effect is partially compensated by the fact that $A$ votes to adopt sometimes. But since A's signals are not good, it is not of much help.
} 


\section{A. Second Opinion}

Suppose that decision maker $D$ is more liberal than expert $A\left(k^{d}<k^{a}\right)$. If $D$ 's information favors adoption, then it is natural for the decision maker to make the adoption decision. Consulting the expert seems both unnecessary, because his own information supports his bias, and risky, because $A$ is relatively prone to rejection. On the other hand, if $D$ 's own information argues against adoption, it seems prudent for $D$ to get a second reading by delegating the decision to $A$.

Formally, consider the following delegation mechanism with adoption control: $D$ delegates the decision to $A$ only if $y^{d}$ falls below some threshold $t_{*}^{d}$, and adopts otherwise. This is equivalent to a procedure in which one vote from either $D$ or $A$ is sufficient to adopt, except that voting is sequential rather than simultaneous. Upon delegation, expert $A$ infers $y^{d}<t^{d}$. and makes the decision on the basis of this inference and his own data $y^{a}$. Thus expert $A$ decides in exactly the same way he would have voted if he were a pivotal voter in a team with unilateral adoption. The optimal decision for $A$ is to adopt if and only if $y^{a} \geq t_{*}^{a}$, where the threshold $t_{\text {. }}^{a}$ satisfies

$$
l^{a}\left(t_{*}^{a}\right) L_{*}^{d}\left(t_{*}^{d}\right)=k^{a} .
$$

Anticipating this, decision maker $D$ sets the threshold $t^{d}$. of delegation according to

$$
l^{d}\left(t_{*}^{d}\right) L_{*}^{a}\left(t_{*}^{a}\right)=k^{d} .
$$

Thus the mechanism with adoption control is formally equivalent to unilateral adoption. ${ }^{15}$ The expected cost to the decision maker in this case is given by

$$
C_{*}^{d}=k_{1}^{d}\left[1-F_{n}^{d}\left(t_{*}^{d}\right) F_{n}^{a}\left(t_{*}^{a}\right)\right]+k_{2}^{d} F_{p}^{d}\left(t_{*}^{d}\right) F_{p}^{a}\left(t_{*}^{a}\right) .
$$

In the symmetric situation in which the decision maker is more conservative than expert $A\left(k^{d}>k^{a}\right)$, the mechanism with rejection control takes the following form: $D$ delegates only if $y^{d}$ exceeds some threshold, and rejects otherwise. This is formally equivalent to unilateral rejection. The threshold of delegation for the decision maker, $t_{* .}^{t}$, and the threshold $t_{.}^{a}$. for expert $A$ satisfy

$$
\begin{aligned}
& l^{d}\left(t_{. .}^{d}\right) L_{* .}^{a}\left(t_{* *}^{a}\right)=k^{d}, \\
& l^{a}\left(t_{*}^{a}\right) L_{* \ldots}^{d}\left(t_{*}^{d}\right)=k^{a} .
\end{aligned}
$$

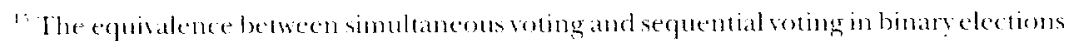

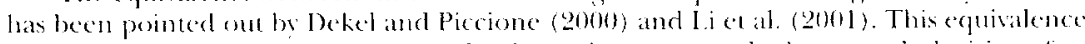
implies that the decision maker neser has incentives to wemule the expent sedecion after delecigation. 
The expected cost to decision maker $D$ in this case is given by

$$
\begin{aligned}
C_{*}^{d} .= & k_{1}^{d}\left[1-F_{n}^{d}\left(t_{* *}^{d}\right)\right]\left[1-F_{n}^{a}\left(t_{* *}^{a}\right)\right] \\
& +k_{2}^{d}\left\{1-\left[1-F_{n}^{d}\left(t_{*}^{d}\right)\right]\left[1-F_{n}^{a}\left(t_{* *}^{a}\right)\right]\right\} .
\end{aligned}
$$

In the case of an uninformed decision maker, when the conflict of interest is sufficiently great between the decision maker and the expert, delegation will never occur. Here, since $D$ can always rely on his own information, the never-clelegate option seems even more attractive. However, we can show that by using the delegation mechanisms, an informed decision maker always benefits by having the option of seeking a second opinion from the expert, regardless of the preference conflict between $D$ and $A$. In particular, $D$ retains control by giving the expert the authority of granting a second chance to adopt a project only if the expert is more conservative than he is. Similarly, he retains control by giving the expert the veto power to kill a project only if the expert is more liberal than he is. Such a combination of delegation and control dominates making the decision without consulting the expert, regardless of the extent of conflicts.

For the following proposition, let $C^{d}$ be the expected cost to the decision maker who never delegates. We have seen earlier that in this case decision maker $D$ uses the threshold $\hat{t}^{d}$, which satisfies $l^{d}\left(\hat{t}^{d}\right)=k^{d}$. Thus

$$
C^{d}=k_{1}^{d}\left[1-F_{n}^{d}\left(\hat{t}^{d}\right)\right]+k_{2}^{d} F_{p}^{d}\left(\hat{t}^{d}\right) .
$$

Proposition 5. If the decision maker is more liberal than the expert $\left(k^{d}<k^{a}\right)$, then the mechanism with adoption control is better than no delegation $\left(C_{*}^{d}<C^{d}\right)$. If the decision maker is more conservative than the expert $\left(k^{d}>k^{a}\right)$, then the mechanism with rejection control is better than no delegation $\left(C_{* *}^{d}<C^{d}\right)$.

The proof is straightforward. Compare $C_{*}^{d}$ and $C^{d}$. When $k^{a}$ becomes arbitrarily large, expert $A$ almost always chooses to reject upon delegation. Since delegation is essentially the same as rejection, decision maker $D$ sets $t^{d}$. very close to $\hat{t}^{d}$. This means that $C_{\text {, }}^{d}$ is arbitrarily close to $C^{d}$. Moreover, since $d C_{*}^{d} / d k^{a}>0$ for all $k^{a}>k^{d}$, we have $C_{*}^{d}<C^{d}$ for all $k^{a}>k^{d}$. By a similar argument, $C_{* .}^{d}<C^{d}$ for all $k^{a}<k^{d}$.

In the case of an uninformed decision maker, we have defined the influence of an expert as the range $\left[L_{*}^{a}\left(\hat{t}^{a}\right), L_{*}^{a}\left(\hat{t}^{a}\right)\right]$ of $D$ 's preference such that delegation occurs. With an informed decision maker, the influence cannot be defined in the same way, since $D$ always benefits from having the delegation option. Instead, we may take $D$ 's threshold in seeking a second opinion as a measure of A's influence. For the delegation mechanism with adoption control, $D$ delegates if $y^{d}<t_{*}^{d}$, so a greater $t_{*}^{d}$ means a more influential expert $A$. Similarly, for the mech- 
anism with rejection control, $D$ delegates if $y^{d}>t_{* *}^{d}$, so a smaller $t_{* *}^{d}$ means a greater influence for $A$.

\section{B. Control}

When the decision maker is a liberal relative to the expert, $D$ wants to retain control by keeping the option of unilateral adoption. The opposite is true when $D$ is a conservative relative to $A$. It turns out that adopting a mechanism that delegates to the expert only when the evidence $y^{d}$ is not conclusive either way accommodates both needs. By retaining the option of making both decisions on the basis of his own information without consulting the expert, the decision maker can reduce manipulation by the expert and increases the value of his expertise.

Formally, suppose that decision maker $D$ delegates the decision to expert $A$ only when he is not sure, that is, when his private evidence is relatively uninformative $\left(y^{d} \in\left[t_{1}^{d}, t_{2}^{d}\right]\right) .{ }^{16}$ Upon delegation, expert $A$ infers that $y^{d} \in\left[t_{1}^{d}, t_{2}^{d}\right]$. Then the optimal decision for $A$ is to adopt if and only if $y^{a} \geq t^{a}$, where the threshold $t^{a}$ satisfies

$$
l^{a}\left(t^{a}\right) \mathcal{L}^{d}\left(t_{2}^{d}, t_{1}^{d}\right)=k^{a} .
$$

Given this, decision maker $D$ chooses to reject if $y^{d}<t_{1}^{d}$, adopt if $y^{d}>$ $t_{2}^{d}$, and delegate if $y^{d} \in\left[t_{1}^{d}, t_{2}^{d}\right]$, where $t_{1}^{d}$ and $t_{2}^{d}$ satisfy

$$
\begin{gathered}
l^{d}\left(t_{1}^{d}\right) L_{* *}^{a}\left(t^{a}\right)=k^{d}, \\
l^{d}\left(t_{2}^{d}\right) L_{*}^{a}\left(t^{a}\right)=k^{d} .
\end{gathered}
$$

The expected cost to $D$ in this case is given by

$$
\begin{aligned}
\tilde{C}^{d}= & k_{1}^{d}\left\{1-F_{n}^{d}\left(t_{2}^{d}\right)+\left[F_{n}^{d}\left(t_{2}^{d}\right)-F_{n}^{d}\left(t_{1}^{d}\right)\right]\left[1-F_{n}^{a}\left(t^{a}\right)\right]\right\} \\
& +k_{2}^{d}\left\{F_{p}^{a}\left(t^{a}\right)\left[F_{p}^{d}\left(t_{2}^{d}\right)-F_{p}^{d}\left(t_{1}^{d}\right)\right]+F_{p}^{d}\left(t_{1}^{d}\right)\right\} .
\end{aligned}
$$

Proposition 6. Delegating the decision with both adoption and rejection controls is better than the delegation mechanisms with only adoption or rejection control $\left(\tilde{C}^{d}<C_{*}^{d}\right.$ if $k^{d}<k^{a}$ and $\tilde{C}^{d}<C_{* *}^{d}$ if $\left.k^{d}>k^{a}\right)$.

We provide only a sketch of the proof of this result; interested readers are referred to a closely related argument in Li et al. (2001). Suppose that $k^{d}<k^{a}$. Delegation only when $y^{d}<t_{*}^{d}$ is equivalent to delegation only when $y^{d} \in\left[y, t_{*}^{d}\right)$, in terms of the expected cost to the decision maker, but the three thresholds $y, t_{*}^{d}$, and $t_{*}^{a}$ are not best responses to each other. Consider an iterative process that begins with the three thresholds

\footnotetext{
${ }^{16}$ An alternative implementation is the following. Decision maker $D$ asks expert $A$ to give an "adopt" or a "reject" recommendation. Decision maker $D$ adopts if and only if $y^{\prime \prime}$ exceeds the threshold $l_{1}^{\prime \prime}$ when $A$ recommends adoption, and he adopts if and only if $y^{\prime \prime}$ excecds a higher threshold tiz when $A$ rccommends rejection.
} 
$\left(y, t_{*}^{d}, t_{*}^{a}\right)$ and converges toward the thresholds $\left(t_{1}^{d}, t_{2}^{d}, t^{a}\right)$. In each iteration the new thresholds are chosen as best responses to the previous thresholds. Fact 4 implies that decision maker $D$ 's two thresholds monotonically increase toward $t_{1}^{d}$ and $t_{2}^{d}$, respectively, whereas expert $A$ 's threshold monotonically decreases toward $t^{a}$. Further, the expected cost to the decision maker falls in each iteration.

Delegating the decision only when his own information is not sure allows the decision maker to retain control of both adoption and rejection decisions, not just the one he is afraid of losing control of. Suppose that $D$ is a liberal relative to $A$. In retaining the option of unilateral adoption, the decision maker anticipates the tendency of $A$ to exaggerate evidence for rejection by using a low threshold of adoption. This has the effect of motivating the expert to manipulate his information and exaggerate the difference between his preferences and those of the decision maker. In contrast, the mechanism with both adoption and rejection controls allows the decision maker to soften his position when he delegates. More information is communicated from $D$ to $A$ in the event of delegation, which reduces the manipulation of the expert and increases the value of his expertise, ultimately benefiting the decision maker.

\section{Conclusion}

As a model of delegation and expertise, the novelty of this paper is to consider an environment in which the value of expertise depends on both how likely delegation occurs and how beneficial delegation is when it does occur, and in which conflicts of interest exist both between the decision makers and the experts and between the experts on a team. Our model allows us to consider a host of issues, including the tradeoff between the influence and the value of experts, the balance of different points of view in an expert team, and the control of the decisionmaking process in delegation. The overall theme of the paper has been the interaction between the need for the decision maker to safeguard own interests and the need to exploit expertise. Controlling the decisionmaking process, through the choice of procedure or through conditioning the delegation decision on the decision maker's own information, turns out to be an effective way for the decision maker to strike a balance between these two opposing needs.

The delegation mechanisms considered in the present paper require the decision maker to specify the game that the experts play upon delegation. At the same time, in order for the decision maker not to have incentives to overrule the decision reached by the expert team, it is important that the decision maker does not monitor how the decision is reached. It is interesting to study how decision makers can balance 
the need to minimize loss of control and the need to make use of expertise in the absence of the commitment to no monitoring and the commitment to not overruling. Another commitment issue worth further research involves paying for expertisc. Organizations often hire external consultants to provide expert opinion on a casc basis. Moncetary incentives to elicit information from self-interested experts have been ignored in our analysis. It can be shown that appropriate ex post moncy transfer mechanisms (after private data are observed) can be used to provide incentives for truthful revelation of private information (Groves 1973; d'Aspremont and Gérard-Varet 1979). However, these mechanisms require ex ante commitment by all parties (bofore private information is observed), which may not be a realistic assumption in a real-life delegation environment. Paying for expertise without ex ante commitment is an interesting issuc to study.

\section{Appendix}

\section{Proof of Proposition 4}

Part i: Since $\partial C_{*}^{\prime} / \partial t_{*}^{u}$ has the same sign as $k^{u}-k^{u}$ and since $d t_{*}^{u} / d k^{\prime}<0$, the first term in equation (6) is negative. Since $C_{*}^{d} / \partial t_{*}^{b}$, has the same sign as $k^{b}-k^{d}$ and since $d t_{*}^{b} / d k^{\prime}>0$, the second term in equation (6) is nonpositive for $k^{\prime \prime} \leq k^{d}$. Thus $d C_{*}^{d} / d k^{b}<0$ for all $k^{b} \leq k^{d}$.

Part ii: Assume that the conditional distributions of $Y^{n}$ and $Y^{h}$ are the same, and drop superscripts $a$ and $b$ for distribution functions and likelihood ratio functions. Let

$$
\Delta=l^{\prime}\left(t_{*}^{a}\right) L_{*}\left(l_{*}^{a}\right) l^{\prime}\left(l_{*}^{b}\right) L_{*}\left(l_{*}^{b}\right)-l\left(l_{*}^{u}\right) L_{*}^{\prime}\left(l_{*}^{u}\right) l\left(l_{*}^{\prime}\right) L_{*}^{\prime}\left(l_{*}^{\prime}\right) .
$$

Since $l / L$, is strictly increasing, we have $\Delta>0$. From equations (2) we can obtain $d t^{\prime \prime} / d k^{b}=-l\left(t_{*}^{\prime}\right) L_{*}^{\prime}\left(l_{*}^{t}\right) / \Delta$ and $d t^{t} / d k^{b}=l^{\prime}\left(t_{*}^{\prime \prime}\right) L_{*}\left(t_{*}^{\prime}\right) / \Delta$. Then we can write $d C_{*}^{d} / d k^{t}$ as

$$
\begin{aligned}
& \frac{\partial C_{*}^{t}}{\partial t_{*}^{\prime}} \frac{d t^{n}}{d k^{\prime \prime}}+\frac{\partial C_{*}^{t}}{\partial t_{*}^{b}} \frac{d t^{b}}{d k^{\prime \prime}} \\
& =\frac{k_{2}^{\prime}}{\Delta}\left[-f_{n}\left(l_{*}^{\prime}\right) F_{n}\left(t_{*}^{b}\right)\left(k^{u}-k^{\prime \prime}\right) l\left(t_{*}^{a}\right) L_{*}^{\prime}\left(l_{*}^{\prime \prime}\right)+\int_{n}\left(l_{*}^{\prime}\right) F_{n}\left(t_{*}^{\prime \prime}\right)\left(k^{b}-k^{\prime \prime}\right) l^{\prime}\left(l_{*}^{\prime \prime}\right) L_{*}\left(l_{*}^{b}\right)\right]
\end{aligned}
$$

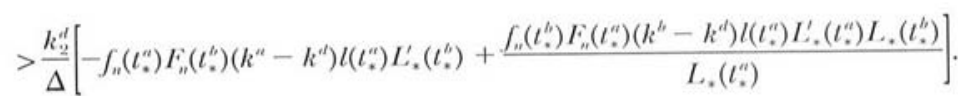

This inequality follows because $l / L$, is increasing. Since $L_{*}^{\prime}=\left(l-L_{*}\right) \int_{n} / F_{n}$, we know that $d C^{d} / d k^{\prime \prime}$ has the same sign as

$$
\begin{aligned}
& -\left(k^{\prime \prime}-k^{\prime \prime}\right) L_{*}\left(l_{*}^{\prime}\right)\left[l\left(l_{*}^{b}\right)-L_{*}\left(l_{*}^{b}\right)\right]+\left(k^{b}-k^{\prime \prime}\right) L_{*}\left(l_{*}^{b}\right)\left[l\left(l_{*}^{\prime}\right)-L_{*}\left(l_{*}^{\prime \prime}\right)\right] \\
& =-\left(k^{a}-k^{\prime}\right)\left[k^{\prime \prime}-L_{*}\left(t_{*}^{\prime \prime}\right) L_{*}\left(t_{*}^{b}\right)\right]+\left(k^{b}-k^{\prime}\right)\left[k^{\prime \prime}-L_{*}\left(t_{*}^{\prime \prime}\right) L_{*}\left(t_{*}^{\prime}\right)\right] \\
& =\left(k^{b}-k^{a}\right)\left[k^{\prime}-L_{*}\left(t_{*}^{a}\right) L_{*}\left(t_{*}^{\prime \prime}\right)\right] .
\end{aligned}
$$

Since $k^{b}>k^{\prime \prime}$ and $k^{\prime \prime}>L_{*}\left(l_{*}^{\prime \prime}\right) L_{*}\left(l_{*}^{b}\right)$, we have $d C_{*}^{\prime} / d k^{\prime \prime}>0$. 


\section{References}

Aghion, Philippe, and Jean Tirole. 1997. "Formal and Real Authority in Organizations." J.P.l. 105 (February): 1-29.

Armstrong, M. 1994. "Delegation and Discretion." Manuscript. Oxford: Oxford Univ.

Austen-Smith, David. 1990. "Information Transmission in Debate." American J. Polit. Sci. 34 (February): 124-52.

Calvert, Randall I. 1985. "The Value of Biased Information: A Rational Choice Model of Political Advice." J. Politics 47 (June): 530-55.

Crawford, Vincent P., and Joel Sobel. 1982. "Strategic Information Transmission." Leonometrica 50 (November): 1431-51.

d'Aspremont, Claude, and Louis-André Gérard-Varet. 1979. "Incentives and Incomplete Information." J. Public Licon. 11 (February): 25-45.

DeGroot, Morris H. 1970. Optimal Statistical Decisions. New York: McGraw-Hill.

Dekel, Eddie, and Michele Piccione. 2000. "Sequential Voting Procedures in Symmetric Binary Elections." J.P.F. 108 (February): 34-55.

Dessein, Wouter. 2002. "Authority and Communication in Organizations." Rev. Licon. Stulies 69 (October): 811-38.

Dewatripont, Mathias, and Jean Tirole. 1999. "Advocates." J.P.E. 107 (February): $1-39$.

Duggan, John, and Cesar Martinelli. 2001. "A Bayesian Model of Voting in Juries." Games and Eron. Behavior 37 (November): 259-94.

Feddersen, Timothy J., and Wolfgang Pesendorfer, 1996. "The Swing Voter's Curse." A.L.R. 86 (June): 408-24.

19. 1997. "Voting Behavior and Information Aggregation in Elections with Private Information." Fconometrica 65 (September): 1029-58.

Gilligan, Thomas W., and Keith Krehbiel. 1987. "Collective Decisionmaking and Standing Committecs: An Informational Rationale for Restrictive Amendment Procedures." J. Latu, Lcon., and Organization 3 (Fall): 287-335. 1989. "Asymmetric Information and Legislative Rules with a Heterogencous Committee." American J. Polit. Sci. 33 (May): 459-90.

Green, Jerry R., and Nancy L. Stokey. 1980. "Two-Person Games of Information Transmission." Manuscript. Cambridge, Mass.: Harvard Univ.

Groves, Theodore. 1973. "Incentives in Teams." Econometrica 41 (July): 617-31.

Holmstrom, Bongt. 1984. "On the Theory of Dclegation." In Bayesian Models in Economic Theory, edited by Marcel Boyer and Richard E. Kihlstrom. Amsterdam: Elsevier Sci.

Krishna, Vijay, and John Morgan. 2000. "Asymmetric Information and Legislative Rules: Some Amendments." Manuscript. Harrisburg: Pennsylvania State Univ.

- 2001. "A Model of Expertise." Q.J.L. 116 (May): 747-75.

Letterie, Wilko, and Otto H. Swank. 1997. "Learning and Signalling by Advisor Selcction." Public (hoice 92 (September): 353-67.

Letterie, Wilko, Oto H. Swank, and Hendrik P. van Dalen. 2000. "When Policy Advisors Cannot Reach a Consensus." Soc. Choice and Welfare 17 (May): 43961 .

I.i, Hao. 2001. "A Theory of Conservatism." J.P.E. 109 (June): 617-36.

Li, Hao, Sherwin Rosen, and Wing Suen. 2001. "Conflicts and Common Interests in Committees." A.F.R. 91 (December): 1478-97.

Melumad, Nahum D., and Toshiyuki Shibano. 1991. "Communication in Settings with No Transfers." Rand J. Licon. 22 (Summer): 173-98.

Meycr, Margaret A. 1991. "Learning from Coarse Information: Biased Contests and Carecr Profiles." Rev. Lcon. Studies 58 (January): 15-41. 
Milgrom, Paul R. 1981. "Good News and Bad News: Representation Theorems and Applications." Bell J. Eicon. 12 (Autumn): 380-91.

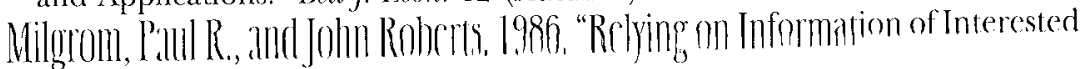
Parties." Rand J. Lcon. 17 (Spring): 18-32.

Ottaviani, Marco, and Peter Sorensen. 1999. "Professional Advice." Discussion Paper in Economics no. 99-04. London: Univ. Coll. London.

Shin, Hyun Song. 1994. "The Burden of Proof in a Game of Persuasion." J. Econ. Theory 64 (October): 253-64. 